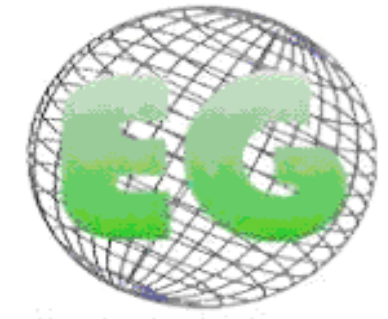

ISSN 1695-6141

N24

www.um.es/egloball

\title{
REVISIONES
}

\section{Historia de la formación del recurso humano de enfermería en Venezuela}

History of the human resource training of nursing in Venezuela

*Rodríguez, Y., **Mejías, M., "**Moreno., M.

*Doctora en Enfermería. Profesora Titular. ${ }^{* *}$ Magister en cuidado integral al adulto críticamente enfermo. Profesora agregada. ${ }^{* * *}$ Magíster en Administración de los servicios en enfermería. Profesora asociada. Universidad de Carabobo. Venezuela.

Palabras clave: Formación; Enfermería; Profesión; Educación; Historia

Keywords: Education; nursing; occupation; education; history

\section{RESUMEN}

La educación en Enfermería ha estado inmersa en el devenir histórico de la sociedad y relacionada con variables: económicas, sociales y políticas que han influido en su desarrollo, por tal motivo para comprender el presente es imperativo volver la mirada al pasado. Este nos muestra que la enfermería ha pasado de ser un oficio a una profesión, pero a diferencia de otras profesiones que desde el comienzo de la universidad se erigen como tales, la enfermería por su parte tuvo que esperar mucho tiempo para poder llegar a las universidades, las cuales surgen en virtud de que las sociedades humanas se tornan más complejas, por lo que se requiere que las personas aprendan una práctica sustentada en una técnica y hasta en una teoría sobre cómo hacerlo, necesitando de métodos para transmitir el conocimiento. Esta necesidad hace que la educación tenga una intencionalidad, sistematización y organización, hecho que influye en la creación de la universidad y posteriormente en la formación de las enfermeras. A continuación se presenta una revisión bibliográfica de la formación de los profesionales en enfermería de la República Bolivariana de Venezuela.

\section{ABSTRACT}

Nursing education has been immersed in the historical development of society and related variables - economic, social and political - that have influenced its development. Thus, to understand the present it is imperative to look to its past. This past shows us that nursing has evolved from a craft to a profession, but unlike other professions which since the beginning of the university have been constructed as such, nursing had to wait a long time to be taught at universities, which arose as human societies become more complex. People are required to learn a practice supported by a technique and even a theory on how to do it, and methods are needed to convey the knowledge. This requirement means that education is intentional, systematic and 
organized, which is influential in the creation of the university and later in the training of nurses. The following is a literature review of training for nurse practitioners of the Bolivarian Republic of Venezuela.

\section{INTRODUCCIÓN}

En Venezuela la primera institución universitaria se funda en 1721 cuando se le concede al Colegio Seminario de Santa Rosa de Lima la facultad de dar grados y erigirse como universidad; posteriormente, en el año 1810 se funda la Universidad de los Andes, para la época estas dos universidades marcarían las pautas de la enseñanza universitaria ${ }^{(1)}$.

Sin embargo, a pesar de su estatus de universidad las mismas seguirían funcionando como seminarios con tradición escolástica, en las cuales se dictaban las cátedras de Filosofía y Sagrada Escritura. Por su parte, la salud estaban en manos de las congregaciones religiosas las cuales fueron mantenidas bajo la jurisdicción de la iglesia ${ }^{(2)}$. El cuidado era ejercido de manera empírica por miembros de organizaciones religiosas, damas de la nobleza criolla, curanderos y familiares.

En el año de 1810, Venezuela logra su independencia de España, en esa época el país era netamente agrícola cuya población vivía azotada tanto por las guerras civiles como por las epidemias. La baja urbanización del país limitaba sensiblemente las posibilidades de cobertura y atención a la comunicación nacional, pues los asentamientos humanos estaban tan distantes que no había una infraestructura vial para facilitar cualquier acción oportuna e integral ${ }^{(3)}$. Es fácil suponer que en materia de servicios como los de salud la población atendida era fundamentalmente la que vivía cerca del médico. Por su parte, la educación era bastante deficiente, solo las clases pudientes tenían este privilegio de acceder a la primaria y secundaria, en cuanto a la educación superior era casi inexistente.

En 1827 el Libertador Simón Bolívar pone en manos del Dr. José María Vargas la dirección de la Universidad de Caracas, quien abre las cátedras de anatomía, cirugía, física experimental y química ${ }^{(4)}$. En esta época comienza el periodo de secularización relacionado con la economía precapitalista de la época, esta separación entre la iglesia y el Estado permite la introducción de las nuevas corrientes científicas y para el año 1891 se abre la Universidad del Zulia y en 1892 la Universidad de Carabobo con carreras como medicina, derecho y educación, quienes hacían otras carreras era porque podían estudiar en el exterior. Sin embargo, la población en su mayoría era analfabeta sobre todo a nivel de los campos, por tanto, el progreso del país fue lento.

En 1899 llega al poder Cipriano Castro por medio de la "Revolución Restauradora", esto trae como consecuencia que las potencias europeas bloquearan los principales puertos venezolanos, lo que llevó al gobierno a crear nuevos impuestos y a rebajar sueldos y salarios, afectando, entre otros servicios, al funcionamiento de las instituciones educativas, en especial, las universidades, además de los conflictos ideológico y científico, que también estaban presentes en aquellos años. La Universidad Central de Venezuela y la Universidad de Los Andes son las únicas universidades del país que quedan en funcionamiento. La universidad en estas primeras décadas, estuvo más en conflictos con el gobierno que en actividad académica y científica plena.

En este año durante la presidencia del Dr. Juan Pablo Rojas Paúl ingresan al país desde Francia las hermanas de San José de Tarbes las cuales realizan trabajos de Enfermería en el Hospital Vargas ${ }^{(5)}$. Estas religiosas modifican la práctica de enfermería de acuerdo a los avances de la época. 
En el año 1907 se inicia la preparación formal de personal de enfermería en el Hospital Ruiz y Páez de Ciudad Bolívar; primer curso para auxiliares con duración de dos años. Posteriormente se crea la Cruz Roja Venezolana cuyo curso de seis meses estaba dirigido a liceístas y damas de la sociedad, quienes eran llamadas Samaritanas. En el año 1912 se crea la Escuela de Artes y Oficios, la cual incluía dentro de sus asignaturas el "Arte de Enfermeras". En este mismo año, se le asigna al Dr. Francisco Antonio Rísquez que elabore un programa señalándose como objetivo la enseñanza teórica- práctica de los conocimientos necesarios para prestar cuidados a los enfermos.

En 1913 se inician los estudios y se implementa el Plan de Estudio elaborado por el Dr. Rísquez y aprobado por el Ministerio de Instrucción, creando en 1913 la Escuela de Enfermería. Esta formación se basaba en un curso teórico - práctico, con duración de dos años, con primaria elemental como requisito de ingreso. Para la época se observa la preocupación por dedicar mayor tiempo a la formación de la enfermería; sin embargo, esta formación estará subordinada a los diseños curriculares del componente médico ya que la Enfermería seguiría viéndose como un oficio destinado a las clases de menor nivel educativo.

En 1914 comienza en Venezuela la explotación petrolera lo que trae al país elevados ingresos económicos y un gran capital humano que influye notablemente en el desarrollo económico, político, social y sanitario del país. La población era atacada por las epidemias principalmente el paludismo, esta enfermedad impedía que las petroleras pudieran desarrollarse porque requerían de mano de obra sana y cualificada para la explotación del petróleo.

En este contexto, en julio de 1915 se promulga la Ley de Instrucción el "Estudio Especial de la Profesión de Enfermería" dando así mayor autonomía a la enseñanza de Enfermería lo que permitió en el periodo de 1919 hasta 1939 la creación de varias escuelas a nivel nacional: Puerto Cabello, San Cristóbal, Maracaibo, Mérida, La Cruz Roja de Ciudad Bolívar, la del hospital Vargas, Hospital de Niños y la del Hospital Luis Razetti, en Caracas ${ }^{(6)}$. A pesar del número de escuelas se evidenció una rivalidad en cuanto a la formación, lo que generó un atraso del proceso de profesionalización.

Las congregaciones religiosas realizaron una encomiable labor dentro de los hospitales, sin embargo, las mismas fueron factores de atraso para la enfermería ya que se opusieron a las pasantías de las estudiantes en los hospitales y también a que las primeras egresadas de Caracas y Maracaibo prestaran sus servicios en los hospitales administrados por ellas, por lo que las enfermeras debieron dedicarse al trabajo a nivel domiciliario ${ }^{(7)}$. Estos problemas originaron el cierre de la Escuela de Enfermeras en el año 1924, iniciándose así un periodo de latencia de casi seis años.

En 1930 llegan al país enfermeras puertorriqueñas quienes hacen gestiones para crear la Escuela de Enfermeras de Caracas, esta escuela no entra en funcionamiento por las barreras existentes, que no permiten su adscripción al Hospital Vargas. Es hasta el año 1934 cuando se logra fundar la Escuela de Enfermeras del Hospital Vargas. En 1935, tras la muerte del Dictador Juan Vicente Gómez surge el interés por la educación técnica de la cual se carecía, sobre todo de maestros y enfermeras.

Para el año 1936, cuando finaliza el período de la dictadura, la salud y la educación se convierte en una de las prioridades del Estado venezolano. Se crea el Ministerio de Sanidad y Asistencia Social, se intensifican los programas de alcance sanitario para combatir las epidemias, encontrándose como obstáculo la escasez de personal preparado. Se crean 
entonces tres escuelas de enfermería: Dr. Francisco Rísquez, Hospital de Niños en Caracas y la Escuela anexa a la Compañía Caribbeam Petroleum en Maracaibo.

Asimismo este ministerio realiza convenios con la Fundación Rockefeller para iniciar programas en nuestro país y crear unidades locales de salud. Este hecho influye notablemente en el desarrollo de enfermería en el país ya que esta fundación trae personal extranjero. Así la enfermería a nivel medio se inicia con la Escuela Normal Profesional de Enfermeras en 1938, cuyo objetivo era formar docentes para futuras escuelas de enfermería en el país; y la Escuela Nacional de Enfermeras (ENE) en 1940.

Conforme se fue desarrollando el proyecto de la escuela, se fue haciendo manifiesta cierta dificultad para conjurar los principios que debían guiar la formación en enfermería, con las prácticas docentes de otras escuelas. Se mantuvo una actitud crítica, innovadora y coherente sobre las directrices en la formación de las enfermeras, no permitiendo que las alumnas hicieran prácticas en hospitales que no se consideraban que pudieran ofrecer una calidad docente mínima.

En tal sentido, los conocimientos teóricos y la práctica estuvieron centrados en la enfermedad apoyados en la bibliografía médica de la época, todas las materias, incluyendo patología y parasitología eran impartidas por clínicos ${ }^{(8)}$. La instrucción teórica y práctica no se correlacionaban, ya que las estudiantes recibían adiestramiento práctico en medicina y cirugía, mientras que las clases sobre esas materias no se darían sino al año siguiente. A las estudiantes se les enseñaba simples hábitos de baño, alimentación y sueño, como cuidados elementales.

La tendencia pedagógica en la enseñanza de la Enfermería era el modelo clásico que se denomina pedagogía de la transmisión que supone que el docente es conocedor de la información y el alumno el desconocedor; por tanto la instructora demostraba los procedimientos y el estudiante redemostraba. En tal sentido, el aprendizaje se demostraba por la capacidad de alumno de realizar los procedimientos como le eran demostrados. Evidentemente el reforzamiento fortalece una respuesta, pero el aprendizaje es más complejo, ya que intervienen diferentes variables, para que este sistema de enseñanza cumpla su finalidad. Por tanto, la actividad práctica es considerada como instrumental donde se demuestran las habilidades y destrezas adquiridas más que la actividad donde se pueda crear el saber y confrontar el referente teórico.

Por otra parte, la evaluación era considerada como el proceso que jerarquiza y vigila la cantidad del trabajo realizado y muy pocas veces la calidad del conocimiento ${ }^{(9)}$. La curricula sigue un orden deductivo, subdividido en disciplinas sin mayor relación entre ellas centrado en los aspectos biomédicos y técnicos.

Posteriormente se pasa al método de enseñanza teórico - práctico en el cual la instructora dictaba clases magistrales, cuya evaluación consistía en exámenes escritos y orales, pruebas prácticas, y asignaciones para valorar la habilidad de la participante, se realizaba en forma individual y periódicamente, con la finalidad de validar el método de enseñanza y la capacidad del alumno. Durante este periodo de formación prevalecía el modelo autoritario el cual tiene sus raíces en: la disciplina como instrumento de vigilancia, la responsabilidad normalizada como parámetro de eficiencia, y la subordinación profesional, análoga a la subordinación como género. Prevalece una marcada tendencia a la obediencia, el respeto a la autoridad y a la norma. El sentido de la autoridad como dominio frente al otro, donde se establecen relaciones de opresor/ oprimido (maestro - alumno) conlleva el reforzamiento de 
la pasividad la que se traduce en sumisión, aspecto que aún en el siglo XXI podrían estar presentes en la formación.

En la formación de profesionales de Enfermería se aplica un enfoque eminentemente biologicista, los planes de estudio se organizan en torno a cuatro especialidades médicas tradicionales: medicina general, cirugía, obstetricia y pediatría, considerándose asignaturas del área tales como técnicas y procedimientos del arte de enfermería, cuidados de enfermos de medicina general y cirugía general. En la formación de Enfermería se hace evidente que el modelo pedagógico se ha fundamentado en el modelo de "aprender haciendo" y en la vigilancia moral y técnica de las aprendices, sistema que surge de la Escuela Nightingale y se transfiere a otras escuelas ${ }^{(10)}$. Este sistema aún en nuestros días pareciera permanecer en el sistema de formación de las enfermeras(os), dando mayor importancia a la práctica y al quehacer diario; a menoscabo del componente teórico que debe fundamental la acción que se realiza, de allí quizás la brecha entre la teoría y la práctica.

Cabe destacar, que en el año 1938 se crea la División de Unidades Sanitarias y al año siguiente se crea la Sección de Enfermeras de Salud Pública lo que origina que en el año 1943 se introduzcan cambios en el pensum de estudios de enfermería, con la incorporación de la signatura "Medicina Preventiva".

En el año 1946, se crea el Instituto de Hospitales adscrito al Ministerio de Sanidad y Asistencia Social y se organiza la Sección de Enfermería Hospitalaria. Para este año, las escuelas de enfermería del país habían revisado el curriculum de formación del personal de enfermería, de manera que las egresadas de las escuelas tuvieran una formación integral y una alta capacidad técnica cónsona con las exigencias de la situación de salud en el país y la demanda de servicios.

Las décadas de los años 40 y 50 se caracterizan por el inicio de la explotación a gran escala de yacimientos petroleros y la conversión de país rural y agrícola en país urbano dependiente casi exclusivamente de ese recurso natural. El cambio fue drástico, se pasa de una Venezuela agraria, fundada en el poder de los caudillos, a otra Venezuela que avanzaba en la urbanización, lo primero fue la ampliación del sistema educativo superior con la fundación del Instituto Pedagógico Nacional en 1936. En este mismo año, se reinician las gestiones para la reapertura de la Universidad del Zulia en 1946.

Los ingresos por el "oro negro" permiten al gobierno ejecutar una política de desarrollo económico basada en la construcción masiva de obras de infraestructura y de industria pesada, en este periodo las universidades establecieron nuevas carreras, sin embargo, en cuestiones de curriculo, mantuvieron un plan de estudios rígido, la enseñanza magistral y la estructura académica.

Para mediados de siglo, una mayor proporción de la población comenzaba a ingresar en la educación superior, se pensaba que para proporcionar mejor atención médica se debía formar enfermeras mejor educadas y esto debía hacerse en una escuela universitaria (11). Aunado a esto, a nivel internacional se crea la Organización Mundial de la Salud (OMS) lo que influye positivamente en el desarrollo de la Enfermería ya que se establece un nuevo concepto de salud, dándole un significado más positivo a ésta y no la mera ausencia de enfermedad, lo que suponía cambio la atención sanitaria. Uno de los objetivos de la OMS es la formación adecuada de personal sanitario, recomendando a los países miembros las características que la formación de enfermeras debe tener y el papel a desarrollar de estos profesionales dentro del sistema sanitario de cada país. Por otra parte, se promocionó la 
educación en enfermería, la cual se orientó al planeamiento institucional y curricular de las escuelas de enfermería, haciendo énfasis en la enfermera en salud pública ${ }^{(12)}$

En 1954 se fundan las dos primeras universidades privadas del país: la Universidad Santa María y la Universidad Católica Andrés Bello. En 1958 se crea la Universidad de Oriente bajo el concepto de universidad experimental. Estos hechos permiten que el sistema de educación universitaria se divida en universidades: autónomas, experimentales y privadas.

Por su parte, en 1958 se fusionan las secciones de Enfermeras de Salud Pública y la Hospitalaria, creándose la División de Enfermeras la cual asume la responsabilidad y autoridad para organizar, dirigir y evaluar los servicios de enfermería, así como la formación básica y postbásica de enfermeras. En este mismo año, a objeto de elevar el nivel de preparación del personal y facilitar la continuidad de los estudios de enfermería, se modifico el pensum de estudios, elevando la carrera a tres años, con primer ciclo de bachillerato y cuatro años de estudio con primer año de bachillerato como requisito de entrada.

El 2 de junio de 1976 se firma entre el Ministerio de Educación y el Ministerio de Sanidad un convenio que establece que los programas de formación básica en enfermería serían desarrollados en los planteles de educación media diversificada y escuelas técnicas del Ministerio de Educación, a fin de intensificar la formación del personal técnico. Sin embargo, en el año 1964 se da paso al inicio de la enfermería a nivel universitario, con la creación de la Escuela de Enfermería en la Universidad de los Andes, otra en la Universidad del Zulia en 1968, en la Universidad de Carabobo en 1972, en la Universidad Central de Venezuela en 1992. De igual forma son creados los Colegios e Institutos Universitarios para la formación de Técnico Superior Universitario en Enfermería ${ }^{(13)}$

Las enfermeras son preparadas a nivel universitario con una formación de 3 años para los Técnicos Superiores Universitarios y 5 años para los Licenciados en Enfermería. Al ingresar enfermería a las universidades los contenidos de los programas, comienzan a adquirir el nombre de "enfermería". Las reformas curriculares son más frecuentes, desde una reforma educativa del país, hasta propuestas originadas tomando en consideración docentes, estudiantes y asesores internacionales, se producen varios diseños de programas de enfermería. Se precisa el concepto del método científico, de resolución de problemas a través de la aplicación del proceso de Enfermería como instrumento de enseñanza en las clínicas. Este proceso incluía la identificación de problemas enfermeros y la decisión de cursos de acción.

A finales de siglo $X X$ surgen nuevos modelos de enseñanza en el área de la educación, en los cuales el estudiante pasa a ser el protagonista, el centro de atención, el cual requiere de mayor participación en su formación, así mismo se evidencia la necesidad de incorporar a los currículos de Enfermería asignaturas relacionadas con las ciencias sociales y humanísticas así como la investigación. Con este modelo se hace necesario que el docente se actualice en cuantas estrategias metodológicas a través de la formación permanente para el dominio de las estrategias de aprendizaje y su adecuación a los objetivos educacionales.

La mayoría de los planes de estudio de pregrado están formados por tres tipos de materias o actividades académicas: (a) materias básicas generales; (b) materias básicas profesionales; (c) materias optativas, que producen cierta preespecialización profesional; (d) pasantías; y un trabajo de grado. A nivel de pregrado se hace énfasis en la enseñanza del desarrollo del hombre en sus diferentes etapas, utilizándose como referencial teórico básico las necesidades humanas propuestas por Abraham Maslow, las cuales han sido las referencias para identificar las necesidades del cuidado ${ }^{(14)}$ 
En tal sentido, se incorporan asignaturas que se organizan para que el estudiante conozca, estudie, interactué y aprenda el cuidado, de la persona en cada una de sus etapas de la vida, en su entorno, dentro de la familia y la sociedad. A partir del desarrollo de las teorías y modelos de enfermería, el proceso de enfermería, los diagnósticos enfermeros y los planes de cuidados; se incorporan nuevos conceptos y contenidos a la estructura de enseñanza ${ }^{(15)}$, generando cambios a la educación y práctica de la disciplina.

Sin embargo, en las instituciones formadoras del recurso en enfermería, aun prevalece el modelo biomédico asentado sobre los conceptos de síntomas, diagnóstico y tratamiento. En tal sentido, el Consejo Internacional de Enfermería (1999) recomienda que los sistemas de formación de enfermería, garanticen que los planes de estudio se actualicen regularmente para satisfacer las necesidades del entorno cambiante, que se apliquen adecuadamente y se aborden las necesidades de formación constante, de acuerdo al contexto histórico de cada país. Por tanto, la formación de enfermería estará orientada y fundamentada en el principio de la persona que es cuidadora por virtud de su humanidad. Para esto es fundamental fortalecer la importancia del conocimiento de la persona como un ser humano total y completo, considerando que cada persona vive el cuidado en una forma única (16)

Al explorar la realidad de la formación de los profesionales de enfermería en Venezuela se percibe que la dimensión técnico científica se magnífica en detrimento de la relación que debería establecer entre el cuidado, los aspectos humanos del individuo e intrínsecos de las enfermeras; los cuales quedan relegados a un segundo plano por diversos factores de índole administrativo y burocráticos que necesitan ser estudiados.

El hombre es un ser pluridimensional; por tanto, debe estudiarse y tratarse como un ser único, holístico y global; es necesario que la enseñanza se fundamente en un modelo creado por y para enfermería el cual enfatice los valores, elementos fundamentales para la promoción del ser, hacer, conocer y convivir. Asimismo es importante que los diseños curriculares introduzcan asignaturas en la cuales el talento artístico de los participantes aflore ya que es este talento el que se requiere para brindar un cuidado efectivo y eficiente.

Esto plantea a la universidad una reorientación, modificación e implementación de estrategias de enseñanza tendientes a aprovechar al máximo las habilidades, para ofrecer y producir de manera didáctica y real los conocimientos necesarios. Este hecho enmarcado en el desarrollo de competencias que permitan hacer frente a las exigencias cognitivas, prácticas y tecnológicas del ambiente laboral y social, tomando como eje central el cuidado.

\section{REFERENCIAS BIBLIOGRÁFICAS}

(1) ROJAS Reinaldo (2005). Historia de la Universidad de Venezuela. Revista historia de la Educación Latinoamericana. Año/Vol 7. Colombia. Pp. 75 - 100.

(2) SOUSA ALEJANDRA (2003). La formación en enfermería y el desarrollo socioeconómico en América Latina 1850 - 1950. Volumen 1. Número 4.

(3) PORTILLO Mazerosky (2007). Características de la Venezuela Agraria. www.monografía.com

(4) GIL F. José (1930). Historia Constitucional de Venezuela. Tomo I. Caracas. Editores Parra León Hermanos. P. 112.

$(5,6)$ MAGDALENO Betty y colbs (1998). Estudio diacrónico de la educación de enfermería en el estado Carabobo periodo 1947 - 1989. Trabajo de investigación. Universidad de Carabobo. Escuela de Enfermería. 
(7) VII CONGRESO VENEZOLANO DE SALUD PÚBLICA (1986). Tomo VI. 25 de febrero al 1 de mayo. Caracas.

(8) VESSURI Heber (1999). Enfermería de salud pública, modernización y cooperación internacional. El proyecto de la Escuela de Enfermeras en Venezuela, 1936 - 1950. Historia, Ciencias, Saúde: Manguinhos. Río de Janeiro. Volumen VIII. N³. Septiembre - Diciembre

(9) ROMERO María (2000). Algunos aspectos del modelo pedagógico de enfermería. Revista Perspectiva: Salud - Enfermedad. Volumen 8. Colombia.

(10) LONG Kathleen (2004). Formación de los profesionales de enfermería. Revista Nursing. Volumen 22. $\mathrm{N}^{\circ} 10$.

(11) Vessuri H. (1999). Ob. Cit.

(12, 13) FAGIN Claire (1989). Enfermeria del siglo XXI en América Latina. Fundación W.K. Kellogg. Publicado en Nacional League for nurses. Universidad de Pennsylvania.

(14) VASQUEZ, Evelyn (2001). Experiencia Integral el concepto de cuidado en el currículo. El Arte y la Ciencia del cuidado. Grupo cuidado. Universidad Nacional de Colombia. Editora Guadalupe. Primera Edición, reimpresión. Colombia.

(15) MERCHAN Nelson (2002). La evolución de los contenidos teóricos - práctico en enfermería médico- quirúrgica en las tres últimas décadas. Revista Enfermería Clínica. Volumen 10. España.

(16) SANABRIA Luis (2002). Los paradigmas como base del pensamiento actual de la profesión de enfermería. Revista Cubana de educación media superior. 16 (4). 